\title{
Risk factors of multi-drug-resistant tuberculosis in Bangladeshi population: a case control study
}

\author{
Flora $\mathrm{MS}^{1}$, Amin $\mathrm{MN}^{2}$, Karim MR ${ }^{1}$, Afroz $\mathrm{S}^{3}$, Islam $\mathrm{S}^{4}$, Alam $\mathrm{A}^{1}$, Hossain $\mathrm{M}^{5}$ \\ ${ }^{l}$ National Institute of Preventive and Social Medicine, Dhaka, ${ }^{2}$ Combined Military Hospital, \\ Chittagong, ${ }^{3}$ Northern International Medical College, Dhaka, ${ }^{4}$ BRAC Health Program, Dhaka, \\ ${ }^{5}$ Combined Military Hospital, Ghatail, Tangail. \\ E-mail:meerflora@yahoo.com
}

\begin{abstract}
Despite success in tuberculosis control, multi-drug resistant tuberculosis in Bangladesh is increasing and currently multi-drug-resistant tuberculosis rate is $3.6 \%$ in new cases and $19 \%$ in re-treatment cases. This study focused on determination of multi-drug-resistant tuberculosis which is warranted for effective prevention strategy. An unmatched case control study was conducted in 2010. Purposively recruited 136 culture-proved multi-drug-resistant- tuberculosis cases and 152 cured tuberculosis patients were interviewed. Associations between exposure and outcome variables were initially tested by $\chi^{2}$-test, t-test. A result was considered significant at $\mathrm{p}$ value $<0.05$. Effects of exposure variables were also assessed after adjusting for other variables by binary logistic regression models. Crude and adjusted Odds Ratio with 95\% Confidence Interval was computed. Younger age $(\mathrm{p}=0.008)$ and, periurban locality $(\mathrm{p}=0.002)$ were associated with multi-drug-resistant tuberculosis. History of contact $(\mathrm{p}<0.001)$ and tuberculosis in the past $(\mathrm{p}<0.001)$ were four and eight times, respectively, more likely to influence multi-drug-resistant tuberculosis. Regularity [Odds Ratio 0.05; 95\% Confidence Interval (0.01 to 0.39$)$ ] and always observation of treatment [Odds Ratio $0.25 ; 95 \%$ Confidence Interval $(0.10$ to 0.61 )], sputum conversion [Odds Ratio 0.02; 95\% Confidence Interval (0.01 to 0.08)] negatively associated with multi-drug-resistant tuberculosis. Gender and socio-economic status did not show any influence. Treatment course and sputum conversion was the best predictors. Like other developing countries adequacy of treatment is the most important exposure variable. Strengthening of control activities might contribute in preventing development of resistance in tuberculosis patients.
\end{abstract}

\section{Introduction}

Despite the availability of effective chemotherapy, tuberculosis (TB) remains a major health problem in most of the countries of the world ${ }^{\mathbf{1}}$. It represents more than a quarter of the world's preventable death $^{2}$. TB has returned in a deadly form called 'multi-drug-resistant tuberculosis' (MDR-TB) ${ }^{\mathbf{3}}$. More than 400,000 cases of MDR-TB emerge every year globally ${ }^{4}$. MDR-TB prevalence in South East Asia is estimated as $2.8 \%$ (95\% CI, 1.9 to 3.6) among new cases and $18.8 \%$ (95\% CI, 13.3 to 24.3) among previously treated cases ${ }^{5}$ and it is estimated to be $1.6 \%-5.2 \%$ in India, $0-21.6 \%$ in Pakistan 6 .

Bangladesh is among the countries with highest TB burden. Proportion of adults infected with TB bacillus is $50 \%$ and estimated TB incidence is 225 per 100,000 populations per year ${ }^{7}$. World Health Organization (WHO) estimated 19\% MDR-TB rate among previously treated cases and $3.6 \%$ among new cases ${ }^{8}$. Recognizing this burden of MDR-TB, Bangladesh government adopted 5 year DOTS plus pilot project. A national guideline has also been developed to manage all registered patients under DOTS-plus project through approved standardized regimen. To diagnose and follow-up of cases a National TB Reference Laboratory has been established and functionalized in National Institute of Diseases of Chest and Hospital (NIDCH) ${ }^{9}$.

MDR-TB is microbial, clinical and programmatic issue. From a microbiological perspective, resistance is caused by a genetic mutation that makes a drug ineffective. An inadequate or poorly administered treatment regimen allows drugresistant mutants to become the dominant strain in a patient infected with $\mathrm{TB}^{\mathbf{1 0}}$. Mainly previous treatment and $\mathrm{TB}$ contact are the strongest determinants of MDR-TB in Burkina Faso, West Africa $^{11}$. MDR-TB patients are more likely to be 
male in Western Europe, where previous treatment was the most important determinant of MDR-TB. In Eastern Europe, where the risk of transmission is greater, male sex was not a risk factor for MDRTB. Although no influence of sex was found on the association between MDR-TB and previous treatment, an effect of sex in modifying the association between previous treatment and MDRTB cannot be excluded. It could be hypothesized that women are more compliant with treatment and therefore less likely to receive inadequate treatment. MDR-TB patients were more likely to be HIV positive, although one study in France and one in Italy did not find any association with this variable, and in a further study the association was limited to new cases. Finally, MDR-TB was associated with being a prisoner in the studies which included prisoners (OR 1.75; 95\% CI 0.90 to 3.40). The estimated risk of MDR-TB for prisoners was studied more frequently in countries of the former Soviet Union than in the rest of Europe ${ }^{\mathbf{1 2}}$.

Except small-scale studies no well-designed study has been done so far for identification of MDR-TB risk factors in Bangladeshi TB patients. Identification of those risk factors might help the policy makers to take next steps in preventing MDR-TB and thus reducing the burden of TB in Bangladesh. This study was aimed to identify the risk factors of multi-drug resistance tuberculosis particularly responsible for Bangladesh situation. Emergence of drug-resistant Mycobacterium tuberculosis (MTB) strains is considered a real threat to achieving tuberculosis (TB) control. The importance of addressing drug resistant $\mathrm{TB}$ is further amplified by more recent reports on extensively drug resistant TB (XDRTB). Furthermore, MDR-TB and XDR-TB require prolonged and expensive chemotherapy, with a decreased cure rate. The study was designed to estimate the contribution of individual risk factors in influencing the development of MDR TB particularly in our context. It was focused to find out the pattern of previous TB treatment and the socio-economic and demographic characteristics of TB patients including MDR TB cases. The study also tried to determine the association of MDR-TB with suspected variables. This assessment of the risk factors in terms of their particular role in developing MDR TB might be useful in order to control them. Thus the study is expected to help policymakers to adopt appropriate preventive measures.

\section{Materials and Methods}

An unmatched case control study was carried out in 2010. The cases were recruited from National
Institute of Diseases of Chest and Hospital, Chest Diseases Hospital, Rajshahi, and TB hospitals at Jalchhatra, Tangail and Shambhuganj, Mymensingh. MDR TB cases were diagnosed by the physicians of the study hospitals, using the definition of WHO 'resistant to the two main first line drugs, Isoniazid and Rifampicin confirmed by culture'. Controls were defined as the cured patients of TB (defined by NTP) ${ }^{\mathbf{1 0}}$, a smear-positive patient who completed entire course of 6(7) and 8(9) months of treatment and sputum smears are negative on at least two occasions i) at the end or during the last month of treatment and ii) on at least one previous occasion, at least one month apart. One control was selected from the cured TB patients for each case from selected DOTS centers in Dhaka city, Jalchhatra and Mymensingh.

At $95 \%$ confidence level and with $90 \%$ power of test, and taking anticipated odds ratio 3.21 estimated sample size was 128 for cases and 128 for controls. During data collection period a total of 136 cases and 152 controls were available and consented to participate and therefore, recruited. Cases and controls were selected purposively from TB registers.

Data were collected by face to face interview and hospital/ treatment records review. Before data collection meetings were organised with health providers of selected clinics for easy running of the study. Six MBBS doctors were recruited for data collection and were trained on sample selection and data collection. Informed written consent was obtained from every respondent and interview was held in private. A pre-tested semi-structured questionnaire in Bangla and checklist were used for data collection. Data included socio-demographic and disease and treatment related data. The sociodemographic data collected were age, sex, locality, educational attainment, and main occupation. Disease related variables included signs and symptoms, bacillary loads, types of TB, contact history and treatment related variables were regimen, category and regularity of treatment, and observation. Ethical approval was obtained from ethical committee of National Institute of Preventive and Social Medicine.

After collection, data were checked thoroughly for consistency and completeness. Statistical Package for Social Sciences (SPSS) version 16.0 for Windows was used to analyze the data. Descriptive statistics were computed and chi-square was carried out to find the association of MDR-TB with qualitative data and t-tests for quantitative data. A result was considered significant at a $\mathrm{p}$ value $<0.05$. Odds ratios (OR) with $95 \%$ CI were calculated. To 
identify risk factors logistic regression models were constructed for controlling the extraneous variables. The variables found significant in univariate analyses were included in the multivariate analysis for the risk factors analysis.

\section{Results}

Cases and controls were almost equally distributed between males and females in a ration of 3:2. Cases $(30.06 \pm 13.59$ years) were, on average, four and a half years younger than the controls $(34.58 \pm 16.26$ years). Younger age groups ( $<40$ years) were more likely to develop MDR-TB than their older counterparts (Table I). The residents of peri-urban area were three times more likely to have MDRTB than their urban and rural counterparts (adjusted OR 3.06 with 95\% CI 1.11 to 8.41). No difference in educational attainment and occupation were noticed between cases and controls. Although higher monthly family income was observed in cases (5857.99 takas) than in controls (5271.51 takas) but the difference was not significant. About two-third of the cases $(61.8 \%)$ lived in kacha house and it was only $2 \%$ higher than the controls. Majority of the controls had smaller family whereas cases were from bigger family $(\mathrm{p}=0.014)$.

Table 1 Socio-demographic characteristics of cases and controls

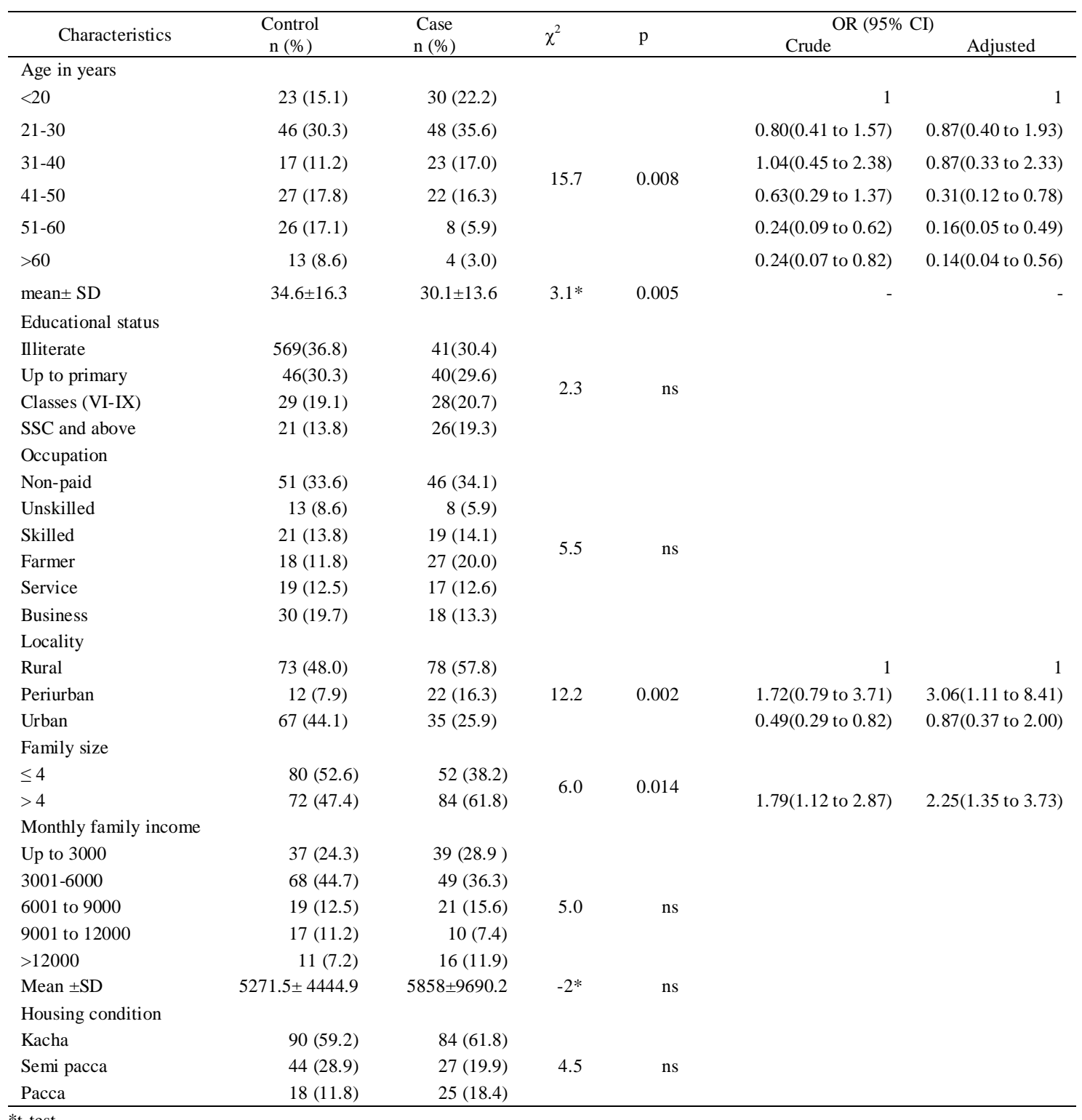


Higher proportion of controls (78.9\%) did not have any history of contact with TB patients than the cases $(57.4 \%, \mathrm{p}<0.001)$. Adjusted odds ratio showed that respondents who had contact history were 3.5 times more likely to have MDR- TB than those who did not have. MDR-TB cases were eleven times more likely to have contact with neighbourhood TB patients than the controls but after adjustment the association disappeared (Table II).

Previous TB treatment related data were presented in table III. Majority started treatment immediately after diagnosis and this proportion was higher among the controls than in cases. Further, if delayed, controls more commonly started treatment within two weeks of diagnosis.

History of tuberculosis in the past was more common in cases $(22.1 \%)$ than in controls $(4.6 \%$, $\mathrm{p}<0.001)$. Adjusted odds ratio showed that previously who had TB were about eight times (OR=5.86; 95\% CI: 2.18- 13.85) more likely to develop MDR- TB than who had not. Cases and controls were equally $\mathrm{BCG}$ vaccinated.

Cases more commonly received category II treatment and after controlling for other variables the influence of treatment type on MDR-TB remained.

More than sixty percent of the cases and controls received treatment from government run DOTS centre, additional $35 \%$ from non-government DOTS centre. However, cases purchased drugs 2.3 times more commonly from medicine shop than the controls $(p=0.037)$. Moreover, cases more frequently changed their treatment place resulting in receipt of drugs from higher number of treatment centres. Cases were two and eleven times more likely to have medicine from two, and three and more centres than the controls. Although the relationship of MDR-TB with treatment place and number of centres disappeared after controlling for socio-demographic variables.

Irregularity in drug intake associated with MDRTB before and after adjustment for other variables. Cases were seven times less likely to have ante-TB drugs regularly. Observation of drug intake also reduced the MDR-TB. Those, who were always observed during treatment, were four times less likely to develop MDR-TB than those who were never observed. Type of observer did not vary between cases and controls.

Sputum conversion after two months showed highly significant association with MDR-TB. Cases were twenty times more likely to be non-converted than the controls and the association remained significant after adjusting for other variables.

A forward logistic regression model was constructed taking the independent variables which showed significant association on uni-variate analyses. The full model was found significant $\left(\chi^{2}=128.36, \mathrm{p}<0.001\right)$ and $87 \%$ of the variation between cases and controls could be explained by the predictor variables. The model could $97.4 \%$ of cases and $93.9 \%$ of controls, and overall $96 \%$ correctly classify. The course of treatment was identified as best predictor followed by sputum conversion.

Table II: Contact with TB patients in cases and controls

\begin{tabular}{|c|c|c|c|c|c|c|}
\hline \multirow{2}{*}{ Contact } & \multirow{2}{*}{$\begin{array}{c}\text { Control } \\
\text { n (\%) }\end{array}$} & \multirow{2}{*}{$\begin{array}{l}\text { Case } \\
\mathrm{n}(\%)\end{array}$} & \multirow{2}{*}{$\chi^{2}$} & \multirow{2}{*}{$\mathrm{p}$} & \multicolumn{2}{|c|}{ Odds Ratio $(95 \% \mathrm{CI})$} \\
\hline & & & & & Crude & Adjusted \\
\hline \multicolumn{7}{|l|}{ Contact } \\
\hline No & $120(78.9)$ & $78(57.4)$ & \multirow{2}{*}{15.6} & \multirow{2}{*}{$<0.001$} & 1 & 1 \\
\hline Yes & $32(21.1)$ & $58(42.6)$ & & & $2.79(1.66$ to 4.68$)$ & $3.43(1.83$ to 6.41$)$ \\
\hline \multicolumn{7}{|c|}{ Relationship with contact } \\
\hline Spouse & $3(9.4)$ & $2(3.4)$ & \multirow{6}{*}{17.3} & \multirow{6}{*}{0.004} & 1 & 1 \\
\hline Parents & $6(18.8)$ & $15(25.9)$ & & & $3.75(0.49$ to 28.39$)$ & 3.31 (0.19 to 56.53 \\
\hline Friends & $3(9.4)$ & $7(12.1)$ & & & $3.50(0.37$ to 32.97$)$ & $11.96(0.42$ to 340.49$)$ \\
\hline Neighbour & $2(6.2)$ & $14(24.1)$ & & & $10.50(1.03$ to 107.17$)$ & 23.89 (0.89 to 642.87$)$ \\
\hline Siblings & $14(43.8)$ & $6(10.3)$ & & & $0.64(0.09$ to 4.89$)$ & 0.35 (0.02 to 8.18$)$ \\
\hline Others & $4(12.5)$ & $14(24.1)$ & & & $5.25(0.64$ to 43.14$)$ & 8.35 (0.38 to 184.73$)$ \\
\hline
\end{tabular}


Table III: Previous illness and treatments of cases and controls

\begin{tabular}{|c|c|c|c|c|c|c|}
\hline Variables & $\begin{array}{c}\text { Control } \\
\mathrm{n}(\%)\end{array}$ & $\begin{array}{c}\text { Case } \\
\mathrm{n}(\%)\end{array}$ & $\chi^{2}$ & $\mathrm{p}$ & OR $(95 \% \mathrm{CI})$ & Adjusted OR (95\% CI) \\
\hline \multicolumn{7}{|c|}{ Duration of symptoms (cough) developed in weeks } \\
\hline Mean & 5.17 & 7.38 & $-2.5^{*}$ & 0.001 & & \\
\hline \multicolumn{7}{|c|}{ Treatment started after diagnosis } \\
\hline Immediately & $115(83.9)$ & $89(83.2)$ & \multirow{3}{*}{3.7} & \multirow{3}{*}{ ns } & & \\
\hline $\begin{array}{l}\text { Within } 2 \\
\text { weeks }\end{array}$ & $20(14.6)$ & $12(11.2)$ & & & & \\
\hline$>2$ weeks & $2(1.5)$ & $6(5.6)$ & & & & \\
\hline \multicolumn{7}{|c|}{ Treatment centre } \\
\hline GOB & $95(62.5)$ & $89(65.4)$ & 0.3 & $\mathrm{~ns}$ & & \\
\hline NGO & $52(34.2)$ & $52(38.2)$ & 0.5 & ns & & \\
\hline Shop & $10(6.6)$ & $19(14.0)$ & 4.3 & 0.037 & $2.31(1.03-5.15)$ & $1.95(0.78-4.89)$ \\
\hline \multicolumn{7}{|c|}{ Number of places } \\
\hline One & $131(86.8)$ & $95(73.1)$ & \multirow{3}{*}{11.1} & \multirow{3}{*}{0.004} & 1 & 1 \\
\hline Two & $19(12.6)$ & $27(20.8)$ & & & $1.96(1.03-3.73)$ & $1.53(0.71-3.33)$ \\
\hline Three & $1(0.7)$ & $8(6.2)$ & & & $11.03(1.36-89.69)$ & $8.69(0.98-77.21)$ \\
\hline \multicolumn{7}{|c|}{ Past history of TB } \\
\hline No & $145(95.4)$ & $106(77.9)$ & \multirow{2}{*}{19.5} & \multirow[t]{2}{*}{$<0.001$} & & \\
\hline Yes & $7(4.6)$ & $30(22.1)$ & & & $5.86(2.18-13.85)$ & $8.03(3.02-21.41)$ \\
\hline \multicolumn{7}{|c|}{ BCG vaccination } \\
\hline No & $77(50.7)$ & $67(49.3)$ & \multirow{2}{*}{0.06} & \multirow[t]{2}{*}{ ns } & & \\
\hline Yes & $75(49.3)$ & $69(50.7)$ & & & & \\
\hline \multicolumn{7}{|c|}{ Course of treatment } \\
\hline Category I & $139(91.4)$ & $17912.5)$ & \multirow{3}{*}{180} & \multirow{2}{*}{$<0.001$} & 1 & 1 \\
\hline Category II & $13(8.6)$ & $119(87.5)$ & & & $74.85(34.92-160.44)$ & $120.59(42.54-341.84)$ \\
\hline \multicolumn{6}{|c|}{ Daily drug intake } & \\
\hline No & $4(2.8)$ & $22(16.5)$ & \multirow{2}{*}{16.6} & \multirow{2}{*}{$<0.001$} & 1 & 1 \\
\hline Yes & $148(97.4)$ & $111(83.5)$ & & & $0.14(0.05-0.41)$ & $0.15(0.05-0.47)$ \\
\hline \multicolumn{7}{|c|}{ Observed treatment } \\
\hline Never & $15(9.9)$ & $24(17.6)$ & \multirow{3}{*}{7.3} & \multirow{3}{*}{0.026} & 1 & 1 \\
\hline Sometimes & $12(7.9)$ & $19(14.0)$ & & & $0.99(0.38-2.61)$ & $0.50(0.15-1.64)$ \\
\hline Always & $124(82.1)$ & $93(68.4)$ & & & $0.47(0.23-0.94)$ & $0.25(0.10-0.61)$ \\
\hline \multicolumn{7}{|l|}{ Observed by } \\
\hline Family & $27(20.1)$ & $30(26.8)$ & \multirow{5}{*}{5.6} & \multirow{4}{*}{ ns } & & \\
\hline Health worker & $78(58.2)$ & $69(61.6)$ & & & & \\
\hline Community & $18(13.4)$ & $10(8.9)$ & & & & \\
\hline GP & $11(8.2)$ & $3(2.7)$ & & & & \\
\hline Sputum conver & & & & & & \\
\hline Converted & $77(86.5)$ & $14(24.1)$ & & & 1 & 1 \\
\hline Not converted & $12(13.5)$ & $44(75.9)$ & 57.9 & $<0.001$ & $20.17(8.57-47.44)$ & $42.54(12.72-142.31)$ \\
\hline
\end{tabular}

\section{Discussion}

A few countries in Eastern Europe and in Southeast Asia are areas of concern for the dissemination of MDR-TB because drug resistant strains may be continuously created and circulated, as suggested by the high percentages of multi-drug resistance among both new and previously treated cases ${ }^{12}$. Bangladesh ranks sixth among 22 high TB Burden countries in the world. Moreover, multi-drug resistant TB is an ominous threat for tuberculosis control $^{13}$. This case control study was designed to determine the factors of multi-drug-resistant tuberculosis. Cases and controls were selected purposively and selection of controls was not based on drug sensitivity test but only on treatment outcomes. Although, it was not planned beforehand the study was accomplished without any significant sex difference.

Previous studies show influence of age on MDR$\mathrm{TB}$ and its factors ${ }^{12,14-18}$. Therefore, the current study did not include age as matching variable. This study also supported previous data showing significant differences $(\mathrm{p}=0.008)$. Likelihood of MDR- TB decreased with the increase in age after the age of 40 years.

Socio-economic factors are the known factors for tuberculosis but there role in MDR-TB is not clear. This study attempted to explore that relationship. Educational status, occupation, average monthly family income and housing status were taken as socio-economic variables and none of them varied between cases and controls. A decrease in proportion of MDR-TB was observed with the improvement in educational status in a study in Thailand ${ }^{\mathbf{1 9}}$ and $41 \%$ of MDR-TB patients in Bangladesh were illiterate but no significant association was observed ${ }^{\mathbf{2 0 , 2 1}}$. Majority of MDRTB in Scotland is unemployed ${ }^{22}$ and in Thailand it is $27 \%{ }^{19}$. In Bangladesh the highest proportion of the MDR-TB cases (61.2\%) were in occupations like agriculture, production and transport. No significant difference was observed in income level between cases and controls in Bangladesh ${ }^{21}$. Lack of financial resource and social security might be a 
risk factor for drug resistance in TB treatment. A study identified substandard housing as risk factors for MDR- $\mathrm{TB}^{17}$. TB patients staying in houses of lower standard might be more vulnerable to develop MDR-TB. Majority of the cases had bigger family which was statistically significant. Many studies have reported that crowded living conditions are among the factors responsible for the development of MDR-TB ${ }^{\mathbf{2 3}}$.

Periurban residents were three times more likely to develop MDR-TB than those who resided in urban and rural area. A similar study in Bangladesh revealed that $\mathrm{TB}$ patients from rural areas were fourteen times more likely to develop MDR-TB than in urban area ${ }^{\mathbf{2 1}}$ probably because the study did not focus peri-urban samples. The service delivery in the DOTs centres of peri-urban areas might need to be reviewed.

About half of the cases and controls showed BCG scar which is not consistent with national coverage as this study only included TB cases. This study like a previous one showed that BCG scar in both MDR TB and non-MDR-TB groups were among the insignificant variables ${ }^{15}$. BCG can not protect those who are already infected or those who could develop TB as a result of re-infection. BCG vaccine is known to prevent the more severe types of $\mathrm{TB}$ such as TB meningitis and milliary TB. However, the efficacy of the vaccine in general ranges from $0 \%$ to $80 \%$. The reasons for this variability are: different types of BCG used in different countries, differences in the strains of Mycobacterium tuberculosis prevailing in different regions, different levels of exposure, etc ${ }^{\mathbf{1 0}}$.

The current study also like number of studies showed that TB contact was the strongest determinants of MDR-TB ${ }^{12,20,24}$. Odds ratio showed cases were 3.4 times more likely to have previous contact history than the controls. Either transmission of MDR strains or selection of single drug resistant strains may have contributed to the increase in the prevalence of MDR-TB in western countries. Infectivity of MDR strains is higher than was initially thought. The prevalence of infection among contacts of MDR-TB cases is similar to the prevalence among contacts of cases without MDRTB. The selection of single drug resistant strains of Mycobacterium tuberculosis is attributable only to inadequate treatment since alternative mechanisms such as the presence of plasmids or other genetic elements has never been demonstrated for $\mathrm{TB}^{25}$.

Mainly previous treatment and TB contact were the strongest determinants of MDR-TB in Burkina Faso, West Africa ${ }^{11}$. Inadequate drug treatment of an individual with TB kills the majority of the bacteria but permits the growth of the small number of resistant organisms within that bacterial population which are arising by spontaneous mutation. A population wholly resistant to a single drug then emerges, and continuing inadequate treatment goes on to select from among this population the small number of organisms which have mutated to have further drug resistance. Resistance to one drug may therefore become resistance to two drugs, and then sequentially to many drugs ${ }^{12}$. A population based study in Georgia revealed that patients who had previously been treated for TB were significantly more likely to have MDR-TB than patients who were newly diagnosed and never previously treated. In multivariate analysis, previous TB treatment was an independent risk factor for the presence of MDR$\mathrm{TB}^{25}$. The current study also revealed that who had TB previously were eight times more likely to develop MDR- TB than those who had TB for the first time. A number of studies revealed that MDRTB cases may thus arise by inadequate treatment of an individual who was initially infected by a fully sensitive strain $^{\mathbf{1 2 , 2 3}}$. The adequacy of treatment measured by course of treatment, delay in starting treatment, regularity of treatment and treatment completion. The course of treatment has been identified as the best predictor of MDR-TB. Those who got category II treatment regimen were more likely to develop MDR-TB. Delay in receiving treatment is one of the causes of development of MDR- TB. In the present study, here immediate treatment was defined according to the NTP guideline $^{\mathbf{1 0}}$ that within 14 days of diagnosis anti-TB treatment should be started. Except only a few, all of the cases and controls started treatment immediately after diagnosis. But delay in diagnosis could not be explored due to recall bias. Only indicator could be assessed was the duration of cough which showed significant longer duration in cases (7.38 weeks), on average, than in controls (5.17 weeks) $[\mathrm{p}=0.001]$.

Significant influence of regular intake of drugs was observed- the cases were 6.6 times less likely to take drug regularly than the controls. Sputum smear conversion, an indicator of successful treatment, showed an association with MDR-TB. Inappropriate treatment resulting in non-conversion of sputum smear showed influence in development of MDR-TB.

Improper use is a result of a number of actions, including administration of improper treatment regimens by health care workers and failure to ensure that patients complete the course of treatmen $^{\mathbf{1 0}}$. The controls were most commonly always supervised during taking of drugs than the 
cases $(\mathrm{p}<0.05)$. A similar study of Bangladeshi sample identified tuberculosis patients who did not remain under directly observed treatment were 3 times more likely to develop multi-drug-resistant tuberculosis (OR 3.21, 95\%CI; 1.59 to 6.5$)^{\mathbf{2 1}}$. Strict implementation of observation during anti-TB therapy and immediate treatment seems to be the most effective method of preventing MDR-TB. Concerned health personnel, in the public as well as in the private sector must adhere meticulously to NTP guidelines. A similar study of Bangladeshi sample revealed that multi-drug-resistance was associated with inadequacy of treatment (OR 2.56, $95 \% \mathrm{CI} ; 2.03$ to 3.23$)^{\mathbf{2 1}}$. Unsuccessful treatment plays a double role with respect to MDR-TB-it can be either a result of or a risk factor for, MDR$\mathrm{TB}^{26}$.

MDR-TB reflects the failure of programs to ensure the complete and reliable cure of $\mathrm{TB}$ patients. Therefore the presence of MDR-TB calls, first, for a thorough review and urgent improvement of basic TB treatment. Unless this is done, any programme to control MDR-TB will fail, because a poor programme can generate MDR-TB much faster than any programme can cure MDR $\mathrm{TB}^{27}$. Many Bangladeshi experts express serious concern about the possibility that MDR-TB rates are higher than official statistics suggests, particularly in light of low level of public awareness about the treatment adherence. The moment the patients start to feel good, they stop treatment ${ }^{28}$. In such a situation, the emerging drug resistance in Bangladesh needs further exploration ${ }^{29}$.

Conclusion: Like other developing countries adequacy of treatment and TB contact history are the most important exposure variables. Strengthening of control activities might contribute in preventing development of resistance in tuberculosis patients.

\section{Acknowledgement}

The researchers acknowledge the financial support of National Tuberculosis Control Program of Bangladesh.

\section{References}

1. Kochi A. The global tuberculosis situation and the new control strategy of the World Health Organization. Tubercle 1991; 72: 1-8.

2. WHO. WHO Report 2007. Global Tuberculosis Control. Surveillance, Planning, Financing. World Health Organization Switzerland: 2007.

3. WHO. Drug and multi drug-resistant tuberculosis (MDR-TB). Frequently asked questions. http://www.who.int/tb/challenges/mdr/faqs [accessed on Apr14, 2008].
4. WHO. Stop TB Partnership. The global MDR-TB and XDR-TB response plan 2007-2008. http://www.who.int [accessed on Apr 02, 2008]

5. SAARC Tuberculosis Center. Tuberculosis in the SAARC Region-an update. Kathmandu, Nepal, SAARC Tuberculosis Center: 2009.

6. Anonymous. Estimates of numbers of individuals with MDR TB. British Medical Bulletin. 73-74(1):17-24.

7. NTP. National Tuberculosis Control in Bangladesh. Annual Report 2007. National Tuberculosis Control Programme, DGHS, MOH\&FW. Dhaka: 2008.

8. WHO. Communicable Diseases, Tuberculosis. TB in South- East- Asia, Country profile-Bangladesh 2009.

9. http://nasmis.dghs.gov.bd/dghs_new/dmdocuments/ Bangladesh. pdf. [Accessed on Dec 27, 2011]

10. NTP. National Guidelines and Operational Manual for Tuberculosis Control. $4^{\text {th }}$ Edition. National Tuberculosis Control Programme, DGHS, MOH\&FW. Dhaka: 2009.

11. Diandé S, Sangaré L, Kouanda $S$ and et al. Risk factors for multi-drug-resistant tuberculosis in four centers in Burkina Faso, West Africa. Microb Drug Resist 2009; 15:217-21.

12. Faustini A, Hall AJ, Perucci CA. Risk factors for multidrug resistant tuberculosis in Europe: a systematic review. Thorax 2006; 61:158-63. doi: 10.1136/ thx.2005.045963)

13. Biswas MS. Acknowledgement. In: NTP, DGHS. Tuberculosis Control in Bangladesh, TB Annual Report 2007. Dhaka.

14. Wahab F, Ashraf S, Khan N, Anwar R, Afridi MZ. Risk factors for multidrug-resistant tuberculosis in patients at tertiary care hospital, Peshwar. J Coll Physicians Surg Pak 2009; 19: 162- 4.

15. Suarez GI, Rosriquez BA, Vidal- Perez JL and et al. Risk factors for multidrug-resistant tuberculosis in a tuberculosis unit in Madrid, Spain. Eur J Clin Microbiol Infect Dis 2009; 28: 325- 30.

16. Law WS, Yew WW, Chiu LC, Kam KM, Chan CK, Leung CC. Risk factors for multidrug- resistant tuberculosis in Hong Kong. Int $\mathbf{J}$ Tuberc Lung Dis 2008; 12: 1065-70.

17. Franke MF, Appleton SC, Bayona J and et al. Risk Factors and Mortality Associated with Default from Multidrug- Resistant Tuberculosis Treatment. Clin Infect Dis 2008; 46: 1844- 51.

18. Espinal MA, Laserson $\mathrm{K}$, Camacho $\mathrm{M}$ and et al. determinants of drug- resistant tuberculosis: analysis of 11 countries. Int J Tuberc Lung Dis 2001; 5: 887- 93.

19. Anunnatsir S, Chetchotisakd P, Wanke C. Factors Associated with Treatment outcomes in Pulmonary Tuberculosis in Northeastern Thailand. Southeast Asian J Trop Med Public Health 2005; 36: 324-30.

20. Rahman MA, Hussain MW, Alam F, Jahan MS. Multidrug Resistant Tuberculosis: Experience in Bogra. Journal of Teachers' Association RMC 2005; 18: 30-6. 
21. Amin MN, Rahman MA, Flora MS, Azad MAK Factors associated with multidrug-resistant tuberculosis. Ibrahim Med Coll J 2009, 3(1): 29-33.

22. Anderson LF, Laurenson IF, Blatchford O, Shakir E, McMenamin J, Johnston F. Trends in Multidrugresistant Tuberculosis in Scotland, 2000-7. Eurosurveillance Reports, Vol. 14, Issue 11; 19 March 2009.

23. Baghaei P, Tabarsi P, Chitsaz E and et al. Risk Factors Associated with Multidrug- esistant Tuberculosis. National Research Institute of Tuberculosis and Lung Disease, Iran. Tanaffos (2009) 8 (3), 17- 21.

24. Banu S, Hossain A, Uddin MKF and et al. Pulmonary tuberculosis and drug resistance in Dhaka Central Jail, the prison in Bangladesh. PLoS One 2010; 5: e10759.

25. Lomtadze N, Aspindzelashvili R, Jangava $\mathrm{M}$ and et al. Prevalence and Risk factors for Multidrug-Resistant
Tuberculosis in Republic of Georgia: A population Based Study. Int J Tuberc Lung Dis 2009; 13: 68- 73.

26. Kodmon C, Niemann S, Lukacs J, Sor E, David S, Somoskovi A. Molecular Epidemiology of DrugResistant Tuberculosis in Hungary. J Clin Microbiol 2006; 44: 4258-61.

27. Freiden TR, Khatri GR. Multidrug-Resistant Tuberculosis. In: Narain JP, editor. Tuberculosis Epidemiology and Control. WHO, Regional Office for South East Asia, New Delhi: 2002.

28. TB policy in Bangladesh 2006. http://www.Soros.org/ health/article-publication [accessed on Apr 14, 2008].

29. Khalequzzaman M, Younus $\mathbf{M}$, Rahim $\mathbf{J}$ and et al. Epidemiology and surveillance of multidrug- resistant tuberculosis in urban and rural areas of Bangladesh. http://www.icddrb.org/pub/publication [accessed on Mar 24, 2008]. 